\title{
RELATIONSHIP BETWEEN REDUCED LUNG FUNCTION AND BODY MASS INDEX IN WOMEN FROM RURAL AREA OF RASULWADI OF SANGLI DISTRICT.
}

\author{
P. M. Patil \\ Department of Zoology, Dr. Patangrao Kadam Mahavidyalaya, Sangli.
}

\begin{abstract}
:
In majority of rural areas biomass fuel such as wood, cow dung and crop residue is easily available. Poor families use these biomass fuels for cooking and heating purposes. Majority of poor families lives in Kutcha type of houses. In kutcha type of houses kitchens are not properly ventilated. Incomplete combustion of biomass fuel release smoke which contains high volume and number of air pollutants such as respirable particulate matter $\mathrm{PM}_{10}, \mathrm{CO}_{\text {, }}$ $\mathrm{NO}_{2}, \mathrm{SO}_{2}$, formaldehyde and other organic compounds. Prolonged exposure to such air born pollutants, have adverse effect on the respiratory system of women which causes reduced lung function. There is strong relation between reduced lung function and Body Mass Index. To study relation between reduced lung function and Body Mass Index, total 100 women were selected from rural area of Sangli District Rasulwadi. Out of 100 women 50 women using chulla and 50 women using LPG were selected. Women using chulla were considered as Subject and women using LPG were considered as Control. All women were underwent spirometery to detect COPD. Spirometric parameter, $\mathrm{FEV}_{1} \%, \mathrm{FVC} \%, \mathrm{FEV}_{1} \% / \mathrm{FVC} \%$ were recorded. Body Mass Index of all women was calculated. Body Mass Index was categoried in four groups (Underweight $<20 \mathrm{~kg} / \mathrm{m}^{2}$, Normal Weight 20.0$25.0 \mathrm{~kg} / \mathrm{m}^{2}$, Overweight $25.0-30.0 \mathrm{~kg} / \mathrm{m}^{2}$, Obese $>30.0 \mathrm{~kg} / \mathrm{m}^{2}$ ) In this study we found that out of 50 women who were exposed to biomass fuel smoke 10 women were suffering from Obstructive type COPD $\left(\mathrm{FEV}_{1} \%<70 \%\right)$. In subject women Body Mass Index in underweightcategory, normal weight category, overweight category and obese category was lower than control group.
\end{abstract}

Key words: $\mathrm{COPD}$, Body Mass Index, $\mathrm{FEV}_{1} \%$ (Forced Expiratory Volume per one second), Forced Expiratory Volume per one second / forced vital capacity, COPD.

\section{INTRODUCTION:}

In India, Majority of women from rural area still uses biomass fuel such as wood, cow dung and crop residue for cooking and heating purpose. (Smith et al, 1996). In rural area most common cause of chronic obstructive disease is the indoor air pollution. For rural women biomass fuel such as wood, cow dung and crop residues are easily available. Rural women from low socio economic status live in kutcha type of houseswhere kitchens are not properly ventilated. Incomplete combustion of biomass fuel releases smoke, which contains high volume and number of air pollutants. Such as $\mathrm{PM}_{10}, \mathrm{CO}$, $\mathrm{NO}_{2}, \quad \mathrm{SO}_{2}$, formaldehyde and other organic compounds prolonged exposure to such air causes COPD.

COPD is the inflammation and swelling of the linings of the air way that leads to narrowing and obstruction of airways.

Combustion of biomass produces a large amount of smoke that spreads into the environment as air pollutants. Exposure to such biomass smoke causes adverse effect on respiratory system. Biomass fuel smoke is the most important risk factor for COPD where indoor ventilation is inefficient (Albalaket al.; 1997, De Koninget al.; 1985).

There is strong relation between COPD and Body Mass Index women with COPD develop chronic cough, dyspnea shortness of breath, weight loss occur in some women with COPD is due to low intake of food and also because of additional energy required for breathing. (Brinnelet al, 2006) In COPD there is loss of body weight, which has a negative impact on quality of life (Brinnelet al, 2006) reduced lung function has effect on the body mass index.

\section{MATERIAL AND METHODS}

Survey of women working in the field (Subject) using chulla in rural area Rasulwadi was done. Information regarding age, type of fuel, year of exposure, hours of exposure was collected.50 women above 35 years of the age using chulla for more than 15 years (Subject) and 50 women not using LPG (Control) were selected. Spirometry was done in 100 women, (50 subject women and 50 control women).Forced expiratory volume per one second $\left(\mathrm{FEV}_{1} \%\right)$. Forced Vital Capacity (FVC\%). $\mathrm{FEV}_{1} \% / \mathrm{FVC} \%$ was recorded. If $\mathrm{FEV}_{1} \%<70 \%$ then there is obstructive type of COPD.

ANTHROPOMETRY: (Ancelet al, 1972) 
- In total 100 women Body mass index was calculated by using the formula BMI $\left(\mathrm{Kg} / \mathrm{m}^{2}\right)$ $=$ Mass $(\mathrm{Kg}) /$ Height $\left.(\mathrm{m})^{2} \mathrm{E}\right)$. (Ancelet al, 1972)

- BMI was categorized into four groups (World Health Organization, 2000) in total 100 women. Underweight $\left(<20 \mathrm{~kg} / \mathrm{m}^{2}\right)$, Normal weight (20.0 -25.0 kg/ m²), Overweight (25.0$\left.30.0 \mathrm{~kg} / \mathrm{m}^{2}\right)$, Obese $\left(>30.0 \mathrm{~kg} / \mathrm{m}^{2}\right)$

STATISTICAL ANALYSIS: Calculated $Z$ test based on null hypothesis: (Gupta and Kappor, 1983)

$$
C a l|z|=\left[\frac{\overline{x_{1}}-\overline{x_{2}}}{\sqrt{\frac{\sigma 1^{2}}{n_{1}}+\frac{\sigma 2^{2}}{n_{2}}}}\right]
$$

$\mathrm{H}_{0}$ : There is no significant difference between control and subject women $\mathrm{FEV}_{1} \%$.

Vs

$\mathrm{H}_{1}$ : There is significant difference between control and subject women $\mathrm{FEV}_{1} \%$.

Cal $|Z|=>$ table $Z=1.96$ at $5 \%$ level of significance. If $Z$ value is greater than table value 1.96 then

$\therefore$ Reject $\mathrm{H}_{0}$

$\therefore$ There is significance difference between control and subject $\mathrm{FEV}_{1} \%$.

\section{RESULT AND DISCUSSION:}

Table No.2 represents the mean values of Age, Weight, Height, BMI of control women are 40.64 Yrs, $55.48 \mathrm{Kg}, 149.30 \mathrm{cms}$ and $24.88 \mathrm{Kg} / \mathrm{m}^{2}$ respectively, while mean values of Age, Weight, Height, BMI of Subject women are 41.66 Yrs, $47.46 \mathrm{Kg}, \quad 154.28 \mathrm{cms}$ and $19.98 \mathrm{Kg} / \mathrm{m}^{2}$ respectively. These values are shown at the base of each column in the Table No.2.The calculated $Z$ values of Age, Weight, Height, BMI based on null hypothesis are at the end of each column in the Table No.2. The calculated $Z$ values of Weight, Height and BMI are respectively $7.87 \mathrm{Kg}$, $9.06 \mathrm{cms}$ and $10.47 \mathrm{Kg} / \mathrm{m}^{2}$. These values are greater than the table values 1.96 . So there is significant difference between $Z$ values of Weight, Height, BMI of control and subject. The calculated $Z$ value of Age is 1.63 Yrs this value is less than table value 1.96. So there is no significant difference between $Z$ value of Age of control and subject.
In this study when we compared the results of spirometry of subject and control we found that there is reduction in lung function parameter $\mathrm{FEV}_{1} \%$ and $\mathrm{FEV}_{1} / \mathrm{FVC} \%$ in subject women than control. In Rasulwadi 10 women were having $\mathrm{FEV}_{1} \%<70 \%$. 10 women had Obstructive type of COPD.

Koksalet al. (2013), Berlin et al. (2014), Aroraet al. (2014) in their study they reported that the lung function parameter $\mathrm{FEV}_{1} \%$ and $\mathrm{FEV}_{1} / \mathrm{FVC} \%$ were significantly lower in women exposed to biomass fuel smoke than control. The reduction in $\mathrm{FEV}_{1} \%$ and $\mathrm{FEV}_{1} / \mathrm{FVC} \%$ may be due to chronic inhalation of toxic substances emitted during biomass combustion leading to inflammatory changes in the bronchi and bronchioles. Our results are similar with study of Koksalet al. (2013), Berlin et al. (2014), Aroraet al. (2014).

In the present study when we compared BMI of subject group with that of control group. We found that in subject group BMI is less than control group. The percentages of underweight and normal weight categories in subject were higher as compared to control while the percentages of overweight and obese categories were lower in subject as compared to control.

According to Sajal (2012), Maryam et al. (2012) in their study they found that BMI in the LPG group was significantly higher than in the group of women using biomass. The subjects using biomass fuel are vulnerable to oxidative stress. Low BMI is the major risk factor in the development of COPD. Weight loss occurs in some women with COPD is due to the low intake of food and also because of additional energy required for breathing, similarly in our investigation we found that, low BMI in subject group as compared to control.

\section{REFRENCES:}

Smith, K.R. (1996): Indoor air pollution in India. ANati Med J India. 9:103-104. 
Albalak, R. (1997): Cultural practices and exposure to particulate pollution from indoor biomass cooking: effect on respiratory health and nutritional status among the Aymara Indians of the Bolivian Highlands. Unpublished Doctoral Dissertation, University of Michigan.

De Koning, H.W., Smith, K.R., Last, J.M. (1985): Biomass fuel combustion and health. Bull World Health Organ. 63:11-26.

Brinnel, Caszo., George, A.D. (2006) : COPD and Nutrition. Lung India. 23:78-81.

Ancel, Keys.,Flaminio, Fidanza., Martti, J., Karvonen., Noboru, Kimura. (1972): Indices of relative weight and obesity. J. Chron Dis. 25:329-343.

Gupta, C., and Kapoor, K. (1983): Fundamentals of mathematics statistics. (8).

Koksal, Hulya., Attila , Saygi., Nesrin, Sariman., Emel, Ahci., Sirin, Yurtlu., Huri, Yilmaz., Yeliz, Duzgun. (2013): Evaluation of clinical and functional parameters in female subjects with Biomass smoke exposure. Respir care. 58(3):424-430.
Berlin, Jeneth., Raj, T. (2014) : Altered lung function test in asymptomatic women using biomass fuel for cooking. Journal of clinical and diagnostic research. 10:BC01-BC03.

Arora, Priya., Gupta, Rajesh., Chopra, Rahul., Gupta, Anupama., Mishra, Neena., Sood, Sushma. (2014): Effect of chronic exposure to biomass fuel smoke on pulmonary function test parameters. Int. J. Res. Med. Sci. 2(4):1488-1494.

Sajal, De. (2012): Body Mass Index Among Patients With Chronic Obstructive Pulmonary Disease. Indian $\mathrm{J}$ PhysiolPharmacol. 56(4) :353-8

Maryam, B. Akor-Dewu., Joseph, O. Ayo., Andrew, R. Collins., M.M. Mabrouk., Alexander, B. Adelaiye., Fatima, L. Ciroma. (2012): Comparative study of haematological and cardiorespiratory parameters in women exposed to biomass or mixed fuels. AIJOCR. 2(8) : 257-263.

Table No. - 1

Data of Spirometry (FEV $\%$, FVC\%, FEV 1 /FVC\%) of Rural Women from RasulwadiExposed to Biomass smoke

\begin{tabular}{|c|c|c|c|c|c|c|c|c|c|c|}
\hline \multirow{2}{*}{$\begin{array}{c}\text { Sr. } \\
\text { No. }\end{array}$} & \multicolumn{9}{|c|}{ CONTROL } & \multicolumn{4}{c|}{ SUBJECT } \\
\cline { 2 - 11 } & Age & $\begin{array}{c}\text { Year } \\
\mathbf{s}\end{array}$ & FEV $_{\mathbf{1}} \%$ & FVC\% & $\begin{array}{c}\text { FEV } / \\
\text { FVC\% }\end{array}$ & Age & $\begin{array}{c}\text { Year } \\
\text { s }\end{array}$ & FEV $_{\mathbf{1}} \%$ & FVC\% & $\begin{array}{c}\text { FEV } / \\
\text { FVC\% }\end{array}$ \\
\hline 1 & 40 & 11 & 98.03 & 91.30 & 88.69 & 41 & 22 & 54.46 & 67.47 & 69.05 \\
\hline 2 & 42 & 22 & 76.32 & 86.26 & 88.47 & 43 & 23 & 61.43 & 77.69 & 66.15 \\
\hline 3 & 44 & 24 & 98.03 & 91.30 & 88.69 & 38 & 18 & 121.33 & 114.67 & 86.26 \\
\hline 4 & 41 & 22 & 121.33 & 114.67 & 86.26 & 45 & 25 & 54.46 & 67.47 & 69.05 \\
\hline 5 & 39 & 12 & 98.03 & 91.30 & 88.69 & 42 & 14 & 87.33 & 58.72 & 79.39 \\
\hline 6 & 44 & 24 & 121.33 & 114.67 & 86.26 & 38 & 20 & 53.37 & 36.36 & 80.56 \\
\hline 7 & 36 & 18 & 98.03 & 91.30 & 88.69 & 37 & 20 & 53.37 & 36.36 & 80.56 \\
\hline 8 & 41 & 23 & 121.33 & 114.67 & 86.26 & 39 & 21 & 53.37 & 36.36 & 80.56 \\
\hline 9 & 37 & 20 & 101.27 & 96.37 & 86.02 & 47 & 24 & 87.58 & 86.26 & 88.47 \\
\hline 10 & 40 & 13 & 101.27 & 96.37 & 86.02 & 44 & 26 & 98.03 & 91.30 & 88.69 \\
\hline 11 & 42 & 11 & 98.03 & 91.30 & 88.69 & 41 & 20 & 98.03 & 91.30 & 88.69 \\
\hline
\end{tabular}




\begin{tabular}{|c|c|c|c|c|c|c|c|c|c|c|}
\hline \multirow{2}{*}{$\begin{array}{l}\text { Sr. } \\
\text { No. }\end{array}$} & \multicolumn{5}{|c|}{ CONTROL } & \multicolumn{5}{|c|}{ SUBJECT } \\
\hline & Age & $\begin{array}{c}\text { Year } \\
\text { s }\end{array}$ & FEV $_{1} \%$ & FVC $\%$ & $\begin{array}{l}\text { FEV }_{1} / \\
\text { FVC\% }^{2}\end{array}$ & Age & $\begin{array}{c}\text { Year } \\
\text { s }\end{array}$ & FEV $_{1} \%$ & FVC\% & $\begin{array}{l}\text { FEV }_{1} / \\
\text { FVC\% }^{2}\end{array}$ \\
\hline 12 & 36 & 18 & 121.33 & 114.67 & 86.26 & 45 & 14 & 61.43 & 77.69 & 66.15 \\
\hline 13 & 39 & 21 & 121.33 & 114.67 & 86.26 & 46 & 25 & 54.46 & 67.47 & 69.05 \\
\hline 14 & 42 & 22 & 78.00 & 61.07 & 77.78 & 44 & 24 & 21.56 & 25.74 & 69.23 \\
\hline 15 & 39 & 23 & 95.54 & 91.43 & 86.16 & 45 & 23 & 51.08 & 83.41 & 83.04 \\
\hline 16 & 41 & 20 & 82.56 & 61.07 & 83.04 & 38 & 18 & 33.08 & 36.36 & 80.56 \\
\hline 17 & 45 & 11 & 101.27 & 96.37 & 86.02 & 40 & 20 & 33.08 & 36.36 & 80.56 \\
\hline 18 & 39 & 20 & 121.33 & 114.67 & 86.26 & 46 & 25 & 33.08 & 36.36 & 80.56 \\
\hline 19 & 42 & 23 & 101.27 & 96.37 & 86.02 & 38 & 20 & 61.43 & 77.69 & 66.15 \\
\hline 20 & 40 & 14 & 121.33 & 114.67 & 86.26 & 42 & 13 & 101.27 & 96.37 & 86.02 \\
\hline 21 & 37 & 20 & 121.33 & 114.67 & 86.26 & 40 & 20 & 21.56 & 25.74 & 69.23 \\
\hline 22 & 39 & 21 & 87.58 & 86.26 & 88.47 & 42 & 21 & 54.46 & 67.47 & 69.05 \\
\hline 23 & 41 & 22 & 98.03 & 91.30 & 88.69 & 46 & 25 & 53.37 & 54.82 & 80.56 \\
\hline 24 & 42 & 12 & 101.27 & 96.37 & 86.02 & 44 & 24 & 53.37 & 36.36 & 80.56 \\
\hline 25 & 44 & 22 & 87.33 & 58.51 & 79.39 & 42 & 23 & 92.79 & 90.32 & 86.16 \\
\hline 26 & 48 & 30 & 92.79 & 91.43 & 86.16 & 39 & 18 & 121.33 & 114.67 & 86.26 \\
\hline 27 & 36 & 17 & 121.33 & 114.67 & 86.26 & 42 & 23 & 33.08 & 36.36 & 80.56 \\
\hline 28 & 45 & 26 & 82.56 & 61.07 & 83.04 & 46 & 27 & 33.08 & 36.36 & 80.56 \\
\hline 29 & 39 & 20 & 87.33 & 90.66 & 79.39 & 40 & 14 & 33.08 & 36.36 & 80.56 \\
\hline 30 & 38 & 13 & 105.18 & 102.62 & 86.38 & 43 & 25 & 33.08 & 36.36 & 80.56 \\
\hline 31 & 40 & 21 & 87.33 & 58.51 & 79.39 & 39 & 20 & 78.40 & 72.15 & 85.96 \\
\hline 32 & 44 & 27 & 83.04 & 61.07 & 83.04 & 49 & 30 & 70.94 & 65.56 & 91.14 \\
\hline 33 & 42 & 23 & 98.03 & 91.30 & 88.69 & 37 & 18 & 51.82 & 72.77 & 83.04 \\
\hline 34 & 38 & 10 & 87.58 & 86.26 & 88.47 & 49 & 25 & 92.79 & 91.43 & 86.16 \\
\hline 35 & 39 & 22 & 98.03 & 91.30 & 88.69 & 41 & 21 & 121.33 & 114.67 & 86.26 \\
\hline 36 & 37 & 20 & 121.33 & 114.67 & 86.26 & 42 & 20 & 87.33 & 58.51 & 79.39 \\
\hline 37 & 40 & 21 & 98.03 & 91.30 & 88.69 & 39 & 20 & 87.58 & 86.26 & 88.47 \\
\hline 38 & 41 & 11 & 121.33 & 114.67 & 86.26 & 40 & 21 & 98.03 & 91.30 & 88.69 \\
\hline 39 & 38 & 20 & 101.27 & 96.37 & 86.02 & 42 & 13 & 98.03 & 91.30 & 88.69 \\
\hline 40 & 39 & 21 & 101.27 & 96.37 & 86.02 & 38 & 20 & 50.70 & 53.92 & 87.27 \\
\hline 41 & 40 & 22 & 98.03 & 91.30 & 88.69 & 45 & 24 & 87.58 & 86.26 & 88.47 \\
\hline 42 & 36 & 12 & 121.33 & 114.67 & 86.26 & 42 & 20 & 101.27 & 96.37 & 86.02 \\
\hline 43 & 39 & 20 & 121.33 & 114.67 & 86.26 & 46 & 23 & 80.42 & 91.43 & 86.16 \\
\hline 44 & 42 & 22 & 79.00 & 61.07 & 78.95 & 40 & 20 & 121.33 & 114.67 & 86.26 \\
\hline 45 & 38 & 20 & 92.31 & 91.43 & 85.71 & 37 & 21 & 81.14 & 70.66 & 83.04 \\
\hline 46 & 46 & 26 & 83.04 & 61.07 & 83.04 & 41 & 21 & 42.74 & 39.34 & 93.46 \\
\hline 47 & 49 & 13 & 101.27 & 96.37 & 86.02 & 38 & 18 & 61.43 & 77.69 & 66.15 \\
\hline 48 & 44 & 27 & 121.33 & 114.67 & 86.26 & 40 & 19 & 92.79 & 91.43 & 86.16 \\
\hline 49 & 38 & 20 & 101.27 & 96.37 & 86.02 & 38 & 18 & 121.33 & 114.67 & 86.26 \\
\hline 50 & 44 & 11 & 121.33 & 114.67 & 86.26 & 37 & 20 & 87.33 & 58.51 & 79.39 \\
\hline Mean & $\begin{array}{c}40.6 \\
4\end{array}$ & $\begin{array}{c}19.2 \\
8\end{array}$ & 101.98 & 94.21 & 85.84 & $\begin{array}{c}41.6 \\
6\end{array}$ & $\begin{array}{c}20.9 \\
4\end{array}$ & 70.34 & 69.50 & 81.18 \\
\hline
\end{tabular}




\begin{tabular}{|c|c|c|c|c|c|c|c|c|c|c|}
\hline \multirow{2}{*}{$\begin{array}{l}\text { Sr. } \\
\text { No. }\end{array}$} & \multicolumn{5}{|c|}{ CONTROL } & \multicolumn{5}{|c|}{ SUBJECT } \\
\hline & Age & $\begin{array}{c}\text { Year } \\
\mathbf{s}\end{array}$ & FEV $_{1} \%$ & FVC\% & $\begin{array}{l}\text { FEV }_{1} / \\
\text { FVC } \%^{2}\end{array}$ & Age & $\begin{array}{c}\text { Year } \\
\text { s }\end{array}$ & FEV $_{1} \%$ & FVC\% & $\begin{array}{l}\text { FEV }_{1 /} \\
\text { FVC } \%^{2}\end{array}$ \\
\hline Var. & 9.19 & $\begin{array}{c}24.9 \\
2\end{array}$ & 206.01 & 316.85 & 7.48 & $\begin{array}{c}10.4 \\
6\end{array}$ & $\begin{array}{c}12.8 \\
2\end{array}$ & 812.78 & $\begin{array}{c}688.2 \\
8\end{array}$ & 55.45 \\
\hline Sqrt & 0.63 & 0.87 & 4.51 & 4.48 & 1.12 & & & & & \\
\hline $\bar{Z}$ & 1.63 & 1.91 & 7.01 & 5.51 & 4.15 & & & & & \\
\hline
\end{tabular}

Table No.2

Body Mass Index of Rural Women exposed to biomass fuel smoke Rasulwadi

\begin{tabular}{|c|c|c|c|c|c|c|c|c|c|c|}
\hline \multirow{2}{*}{$\begin{array}{l}\text { SR. } \\
\text { NO. }\end{array}$} & \multicolumn{5}{|c|}{ Control } & \multicolumn{5}{|c|}{ Subject } \\
\hline & Name & $\begin{array}{l}\text { Age } \\
\text { (Yrs) }\end{array}$ & $\begin{array}{c}\text { Weight } \\
\text { (Kg) }\end{array}$ & $\begin{array}{c}\text { Height } \\
\text { (cms) }\end{array}$ & $\begin{array}{c}\text { BMI } \\
\mathrm{Kg} / \mathrm{m}^{2}\end{array}$ & Name & $\begin{array}{l}\text { Age } \\
\text { (Yrs) }\end{array}$ & $\begin{array}{c}\text { Weight } \\
\text { (Kg) }\end{array}$ & $\begin{array}{c}\text { Height } \\
\text { (cms) }\end{array}$ & $\begin{array}{c}\text { BMI } \\
\mathrm{Kg} / \mathrm{m}^{2}\end{array}$ \\
\hline 1 & RRP & 40 & 50 & 150 & 22.22 & YBJ & 41 & 45 & 152 & 19.48 \\
\hline 2 & SBP & 42 & 55 & 148 & 25.11 & $\mathrm{RBJ}$ & 43 & 42 & 153 & 17.94 \\
\hline 3 & ISP & 44 & 49 & 150 & 21.78 & SAJ & 38 & 52 & 150 & 23.11 \\
\hline 4 & RSP & 41 & 51 & 146 & 23.93 & SVJ & 45 & 42 & 154 & 17.71 \\
\hline 5 & HPP & 39 & 50 & 150 & 22.22 & PSJ & 42 & 45 & 150 & 20.00 \\
\hline 6 & IBP & 44 & 52 & 151 & 22.81 & ASJ & 38 & 43 & 152 & 18.61 \\
\hline 7 & SYB & 36 & 59 & 148 & 26.94 & BRJ & 37 & 48 & 158 & 19.23 \\
\hline 8 & SSB & 41 & 55 & 147 & 25.45 & KHJ & 39 & 44 & 155 & 18.31 \\
\hline 9 & SSS & 37 & 58 & 150 & 25.78 & LGJ & 47 & 48 & 152 & 20.78 \\
\hline 10 & KSS & 40 & 52 & 151 & 22.81 & SDJ & 44 & 51 & 152 & 22.07 \\
\hline 11 & SVS & 42 & 56 & 150 & 24.89 & PVJ & 41 & 53 & 155 & 22.06 \\
\hline 12 & SBS & 36 & 50 & 147 & 23.14 & JNB & 45 & 45 & 154 & 18.97 \\
\hline 13 & SVS & 39 & 59 & 149 & 26.58 & SDJ & 46 & 46 & 158 & 18.43 \\
\hline 14 & $\mathrm{SSC}$ & 42 & 57 & 148 & 26.02 & VTJ & 44 & 44 & 160 & 17.19 \\
\hline 15 & SMP & 39 & 58 & 150 & 25.78 & SBC & 45 & 52 & 152 & 22.51 \\
\hline 16 & SPP & 41 & 55 & 149 & 24.77 & JSJ & 38 & 45 & 154 & 18.97 \\
\hline 17 & JRP & 45 & 52 & 148 & 23.74 & KVJ & 40 & 45 & 159 & 17.80 \\
\hline 18 & SVP & 39 & 65 & 150 & 28.89 & PVJ & 46 & 44 & 154 & 18.55 \\
\hline 19 & MBP & 42 & 54 & 147 & 24.99 & JNJ & 38 & 47 & 154 & 19.82 \\
\hline 20 & SJP & 40 & 58 & 151 & 25.44 & UAJ & 42 & 49 & 156 & 20.13 \\
\hline 21 & KBP & 37 & 60 & 149 & 27.03 & LYJ & 40 & 41 & 158 & 16.42 \\
\hline 22 & SMP & 39 & 45 & 146 & 21.11 & BSJ & 42 & 45 & 159 & 17.80 \\
\hline 23 & TSP & 41 & 60 & 152 & 25.97 & SGP & 46 & 46 & 156 & 18.90 \\
\hline 24 & SKS & 42 & 59 & 151 & 25.88 & ASP & 44 & 47 & 159 & 18.59 \\
\hline 25 & SDM & 44 & 69 & 150 & 30.67 & RRJ & 42 & 51 & 152 & 22.07 \\
\hline 26 & MVM & 48 & 61 & 149 & 27.48 & SSJ & 39 & 50 & 152 & 21.64 \\
\hline 27 & UJM & 36 & 62 & 148 & 28.31 & KAJ & 42 & 47 & 158 & 18.83 \\
\hline 28 & SDP & 45 & 60 & 150 & 26.67 & GEC & 46 & 46 & 160 & 17.97 \\
\hline 29 & PVP & 39 & 56 & 149 & 25.22 & SNC & 40 & 48 & 156 & 19.72 \\
\hline
\end{tabular}




\begin{tabular}{|c|c|c|c|c|c|c|c|c|c|c|}
\hline \multirow{2}{*}{$\begin{array}{l}\text { SR. } \\
\text { NO. }\end{array}$} & \multicolumn{5}{|c|}{ Control } & \multicolumn{5}{|c|}{ Subject } \\
\hline & Name & $\begin{array}{l}\text { Age } \\
\text { (Yrs) }\end{array}$ & $\begin{array}{c}\text { Weight } \\
\text { (Kg) }\end{array}$ & $\begin{array}{c}\text { Height } \\
\text { (cms) }\end{array}$ & $\begin{array}{c}\text { BMI } \\
\mathrm{Kg} / \mathrm{m}^{2}\end{array}$ & Name & $\begin{array}{l}\text { Age } \\
\text { (Yrs) }\end{array}$ & $\begin{array}{c}\text { Weight } \\
\text { (Kg) }\end{array}$ & $\begin{array}{c}\text { Height } \\
\text { (cms) }\end{array}$ & $\begin{array}{c}\text { BMI } \\
\mathrm{Kg} / \mathrm{m}^{2}\end{array}$ \\
\hline 30 & SVP & 38 & 65 & 150 & 28.89 & SPJ & 43 & 45 & 158 & 18.03 \\
\hline 31 & RVS & 40 & 60 & 147 & 27.77 & PPJ & 39 & 45 & 160 & 17.58 \\
\hline 32 & CAS & 44 & 58 & 150 & 25.78 & LSJ & 49 & 44 & 152 & 19.04 \\
\hline 33 & MPK & 42 & 55 & 157 & 22.31 & AMJ & 37 & 49 & 155 & 20.40 \\
\hline 34 & SDP & 38 & 62 & 150 & 27.56 & VKJ & 49 & 52 & 151 & 22.81 \\
\hline 35 & MSS & 39 & 59 & 151 & 25.88 & SRJ & 41 & 51 & 153 & 21.79 \\
\hline 36 & AVP & 37 & 68 & 148 & 31.04 & CAJ & 42 & 50 & 151 & 21.93 \\
\hline 37 & SSP & 40 & 50 & 147 & 23.14 & SVJ & 39 & 52 & 158 & 20.83 \\
\hline 38 & KBP & 41 & 65 & 152 & 28.13 & MKJ & 40 & 50 & 154 & 21.08 \\
\hline 39 & SRP & 38 & 63 & 149 & 28.38 & SNJ & 42 & 50 & 154 & 21.08 \\
\hline 40 & CAS & 39 & 60 & 150 & 26.67 & JNJ & 38 & 46 & 152 & 19.91 \\
\hline 41 & PSP & 40 & 51 & 151 & 22.37 & SBJ & 45 & 50 & 156 & 20.55 \\
\hline 42 & SSP & 36 & 58 & 147 & 26.84 & $\mathrm{BDC}$ & 42 & 51 & 158 & 20.43 \\
\hline 43 & MDP & 39 & 45 & 148 & 20.54 & SSM & 46 & 50 & 151 & 21.93 \\
\hline 44 & PTP & 42 & 50 & 149 & 22.52 & $\mathrm{BBJ}$ & 40 & 52 & 146 & 24.39 \\
\hline 45 & SNP & 38 & 43 & 150 & 19.11 & YAC & 37 & 50 & 152 & 21.64 \\
\hline 46 & SPP & 46 & 45 & 147 & 20.82 & RAM & 41 & 49 & 158 & 19.63 \\
\hline 47 & SDP & 49 & 51 & 151 & 22.37 & $\mathrm{ADL}$ & 38 & 40 & 148 & 18.26 \\
\hline 48 & VMP & 44 & 49 & 150 & 21.78 & LSK & 40 & 54 & 154 & 22.77 \\
\hline 49 & MSP & 38 & 48 & 151 & 21.05 & PMK & 38 & 47 & 149 & 21.17 \\
\hline 50 & $\mathrm{BDP}$ & 44 & 42 & 146 & 19.70 & ASM & 37 & 45 & 150 & 20.00 \\
\hline & Mean & 40.64 & 55.48 & 149.30 & 24.88 & & 41.66 & 47.46 & 154.28 & 19.98 \\
\hline & Var. & 9.19 & 40.53 & 3.73 & 7.79 & & 10.46 & 11.37 & 11.36 & 3.20 \\
\hline & Sqrt & 0.63 & 1.02 & 0.55 & 0.47 & & & & & \\
\hline & $\mathbf{Z}$ & 1.63 & 7.87 & 9.06 & 10.47 & & & & & \\
\hline
\end{tabular}

Table No. 3

Weight Category wise Body Mass Index in Control and Subject

womenRasulwadi.

\begin{tabular}{|c|c|c|c|c|c|c|c|c|}
\hline \multirow[b]{2}{*}{ Village } & \multicolumn{4}{|c|}{ Control } & \multicolumn{4}{|c|}{ Subject } \\
\hline & $\begin{array}{c}<20 \\
\mathrm{Kg} / \mathrm{m}^{2}\end{array}$ & $\begin{array}{c}>20.1 \\
\text { and } \\
<24.99 \\
\mathrm{Kg} / \mathrm{m}^{2}\end{array}$ & $\begin{array}{l}>25 \text { and } \\
<29.99 \\
\mathrm{Kg} / \mathrm{m}^{2}\end{array}$ & $\begin{array}{c}>30 \\
\mathrm{Kg} / \mathrm{m}^{2}\end{array}$ & $\begin{array}{c}<20 \\
\mathrm{Kg} / \mathrm{m}^{2}\end{array}$ & $\begin{array}{c}>20.1 \\
\text { and } \\
<24.99 \\
\mathrm{Kg} / \mathrm{m}^{2}\end{array}$ & $\begin{array}{l}>25 \text { and } \\
<29.99 \\
\mathrm{Kg} / \mathrm{m}^{2}\end{array}$ & $\begin{array}{c}>30 \\
\mathrm{Kg} / \mathrm{m}^{2}\end{array}$ \\
\hline Rasulwadi & 2 & 21 & 25 & 2 & 26 & 24 & 0 & 0 \\
\hline
\end{tabular}

